\title{
Identification of Electron and Hole Ionization Rates in GaAs with reference to IMPATT Diode
}

\author{
J. Pradhan ${ }^{1}$, S.K. Swain ${ }^{1}$, S.R. Pattanaik ${ }^{2}$ and G.N. Dash ${ }^{1}$. \\ ${ }^{1}$ School of Physics, Sambalpur University, Jyoti Vihar, Sambalpur 768019, Odisha, India \\ ${ }^{2}$ Apex Institute of Technology and Management, Bhubaneswar 751001, Odisha, India
}

\begin{abstract}
A computer aided simulation study of electron and hole ionization rates in GaAs with reference to the Terahertz source of power called IMPATT diode is presented in this paper. It is based on the breakdown voltage calculation using the computer simulation method developed for the DC analysis of an IMPATT diode. The comparison of simulation results reveals that the break down voltage calculated using carrier ionization rates data reported by Pearsall et al is in good agreement with the experimental reports.
\end{abstract}

Keywords - GaAs, IMPATT, Ionization rates

\section{INTRODUCTION}

GaAs has been considered as an interesting semiconductor material for many solid state devices. It has also been a promising material for realization of IMPact ionization Avalanche Transit Time (IMPATT) diode which has proved itself as a powerful solid state source of microwave power. IMPATT diode can generate more power at mm-wave and Terahertz frequencies compared to other solid state sources. It can also produce higher conversion efficiencies both in the pulsed and continuous wave operating mode and has established as a better performer, than other solid state power devices.

Owing to the excellent material and electrical properties of GaAs as compared to $\mathrm{Ge}$ and $\mathrm{Si}$, it emerged as an attractive semiconductor material for the fabrication of high power and high frequency solid state avalanche device. Due to negative differential electron mobility, GaAs IMPATT diodes achieve higher DC-to$\mathrm{RF}$ conversion efficiency than Si diodes at low frequencies. GaAs IMPATT diodes have demonstrated noise performance comparable to Gunn diodes together with higher power capabilities [1]. It is also an excellent candidate for low noise local oscillators in the submillimeter-wave region [2]. Due to higher ionization rate of GaAs in the avalanche region, it is considered as the base material to study the microwave and noise behavior of GaAs $\sim \mathrm{Ga}_{0.52} \mathrm{In}_{0.48} \mathrm{P}$ IMPATT diodes [3]. It is also observed that GaAs IMPATT diode yields lower values of series resistance and higher values of negative conductance than its Si counterpart at X band [4]. A computer aided study on mm-wave performance of GaAs double drift IMPATT diode for frequencies of operation in the range of 35 to $140 \mathrm{GHz}$ indicates that the values of device efficiency and value of diode negative resistance remains high for GaAs diodes [5].

The ionization rates of electrons and holes play important role in deciding its performance. The knowledge of correct impact ionization rate data is important in the simulation of IMPATT diodes as well as for avalanche photodiodes. Many researchers have reported these data following different methods either experimental or theoretical [6-14]. In fact, there has been a wide range of variation in the reported data at different electric field values. At some value of the electric field, these data come close to each other but at low and high fields their values differ significantly. In view of the considerable difference in the ionization rate values given by different authors, in this paper the authors have taken an interest to find out most appropriate ionization rate data based on the breakdown voltage calculation. The calculated breakdown voltages will be compared with the experimental reports [15] in order to come to an agreement.

\section{REPORTS OF IONIZATION RATES IN GaAs}

The impact ionization in GaAs has been extensively studied and the ionization rates for electrons and holes have been reported as equal and unequal depending on the design of diode. The newly published results differ from the data already reported and at times they are contrary to the prevailing trend that the ionization rates for electrons and holes should be nearly equal at high electric field. Impact ionization is characterized by two coefficients $\alpha$ and $\beta$ for electrons and holes respectively. 

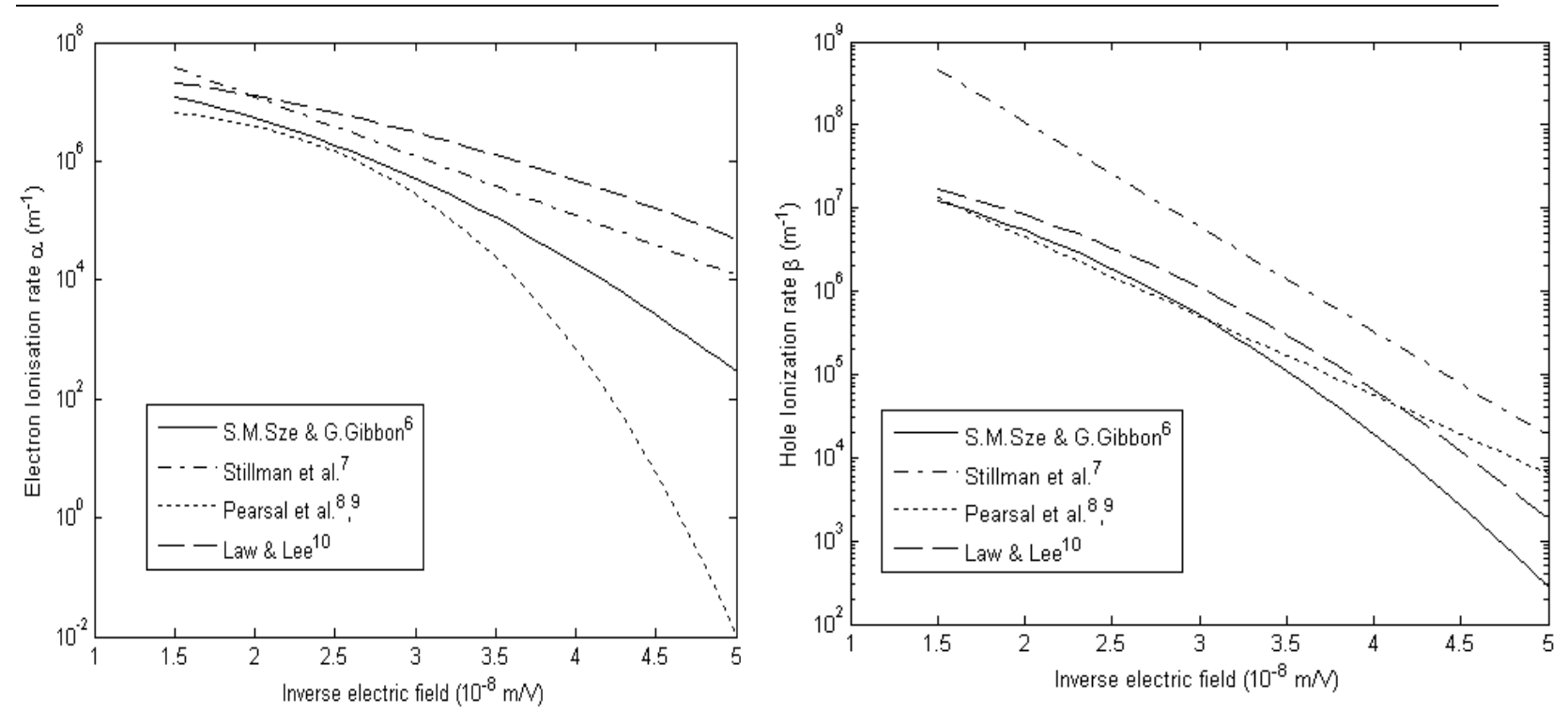

Fig-1 Electron and Hole ionization rate data as a function of inverse electric field for Ref. [6 - 10]

The most accurate method of determining these parameters involves the analysis of photo multiplication data produced under pure electron and pure hole injection. The earliest measurements of ionization parameters in GaAs were inaccurate because the structures were not designed to permit pure injection of one type of carrier. Logan et al. in 1962[16] performed the measurements in diffused $\mathrm{p}^{+} \mathrm{n}$ junctions under electron injection only and assumed that $\alpha=\beta$ in the data analysis. Logan and Sze[17] fabricated complimentary diffused $\mathrm{p}^{+} \mathrm{n}$ and $\mathrm{n}^{+} \mathrm{p}$ junctions, and measured the photocurrent multiplication when either strongly absorbed or penetrating radiation was focused on the top of either structures. This illumination created a pure injection condition and a mixed injection condition, respectively, in the structures and observed that $\alpha=\beta$. Using same phenomenon S.M.Sze and Gibbon [6] also determine the same relation between ionization rates of electron and hole i.e. $\alpha=\beta$.

The experiment showing unequal ionization rates in GaAs were performed by Stillman et al.[7], following the work of Wood et al. [18] in Si, on specially fabricated Schottky-barrier diodes that permitted separate injection of holes and electrons and reported $\beta>\alpha$. Again, at room temperature in $\mathrm{p}^{+} \mathrm{n}$ junction of (100) oriented GaAs, Pearsall et al. $[8,9]$ reported that $\beta>\alpha$. They further observed that the ratio $\beta / \alpha$ decreases at high electric field. In a related work, Capasso et al. $[19,20]$ observed a temperature variation of $\beta / \alpha$ in GaAs with $\beta>\alpha$ below $200^{\circ} \mathrm{C}$ and $\beta<\alpha$ above $200^{\circ} \mathrm{C}$. Law and Lee [10] also performed room temperature measurements on Schottky-barrier diodes and found $\beta>\alpha$ in heavily doped and $\beta<\alpha$ in lightly doped diodes. The ionization rates plots with inverse electric field have been shown in Fig.1.
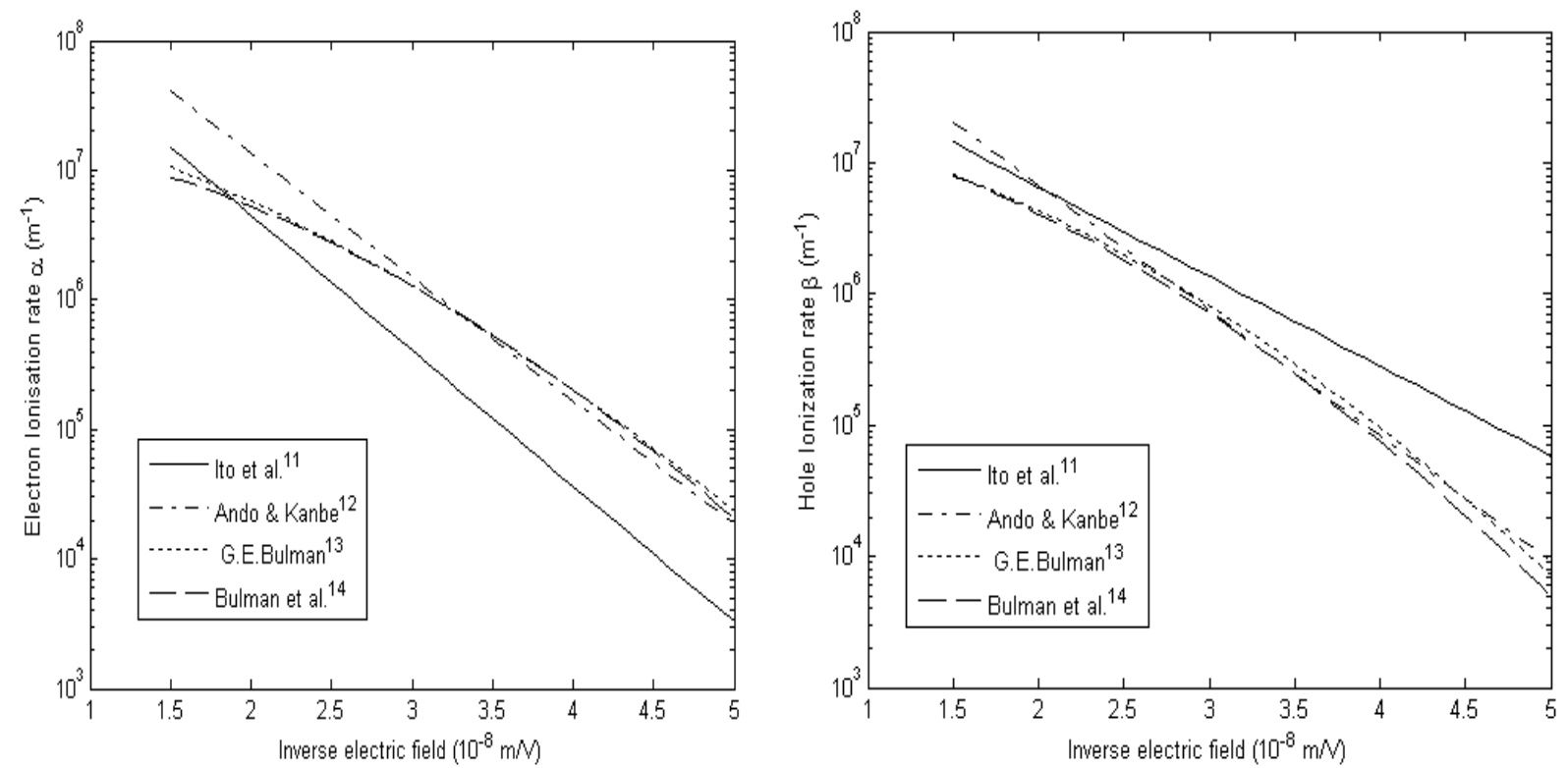

Fig.2 Electron and Hole ionization rate data as a function of inverse electric field for Ref. [11 - 14] 
Ito et al. [11], using a crater mesa $\mathrm{p}^{+} \mathrm{n}$ structure, also observed $\beta>\alpha$, but found that the ratio is strongly field dependent becoming unity at the maximum field. Ando and Kanbe [12] performed photocurrent multiplication and noise measurements for electron injection in a $\mathrm{p}^{+} \mathrm{n}$ junction and found the ratio $(\alpha / \beta)=2$. G.E.Bulman et al. [13], at room temperature, determined electron and hole impact ionization coefficients $\alpha$ and $\beta$ respectively, in (100) oriented GaAs using photocurrent multiplication measurements on specially fabricated p-n junction devices with net donor concentration ranging from $n=1.1 \times 10^{17}$ to $2.2 \times 10^{17} \mathrm{~cm}^{-3}$. Bulman et al. [14] have reported a new set of ionization rate data for GaAs in 1985. The ionization rates of electros and holes reported by Ito et al, Ando and Kanbe, G.E.Bulman and Bulman et al are plotted in Fig. 2. In contrast with many previous reports the electron impact ionization rate is found to be greater than the hole impact ionization rate over the entire doping and electric field range studied. The coefficients of ionization rates of electrons and holes reported by the above researchers have been summarized in Table I.

Table I. Coefficients for ionization rate data published by different authors which have been used in our simulation process.

\begin{tabular}{|c|c|c|c|c|c|c|c|}
\hline \multirow[t]{2}{*}{ References } & \multirow{2}{*}{$\begin{array}{l}\text { Field range } \\
\times 10^{7} \mathrm{Vm}^{-1}\end{array}$} & \multicolumn{3}{|c|}{$\alpha=A_{1} \exp -\left(B_{1 / E}\right)^{\gamma \mathrm{s}}$} & \multicolumn{3}{|c|}{$\beta=A_{2} \exp -\left(B_{2 /}\right)^{x}$} \\
\hline & & $\begin{array}{l}A_{1} \\
\times 10^{8} \mathrm{~m}^{-1} \\
\end{array}$ & $\begin{array}{l}B_{1} \\
\mathrm{x} 10^{8} \mathrm{Vm}^{-1}\end{array}$ & $m$ & $\begin{array}{l}A_{2} \\
\times 10^{8} \mathrm{~m}^{-1} \\
\end{array}$ & $\begin{array}{l}B_{2} \\
\times 10^{8} \mathrm{Vm}^{-1}\end{array}$ & $n$ \\
\hline $\mathrm{Sze}_{6}$ and Gibbons & - & 0.35 & 0.685 & 2 & 0.35 & 0.685 & 2 \\
\hline Stillman et al. ${ }^{7}$ & $2.5-3.3$ & 0.12 & 2.3 & 1 & 3.6 & 2.9 & 1 \\
\hline Pearsall et al. ${ }^{8,9}$ & $3.1-4.76$ & 0.0912 & 0.477 & 3.48 & 3.47 & 2.18 & 1 \\
\hline Law and Lee ${ }^{10}$ & $2.2-4.7$ & 0.454 & 0.628 & 2 & 0.410 & 0.634 & 2 \\
\hline Ito et al. ${ }^{11}$ & $4-6$ & 5.6 & 2.41 & 1 & 1.5 & 1.57 & 1 \\
\hline $\begin{array}{l}\text { Ando and } \\
\text { Kanbe }^{12} \\
\end{array}$ & $0.24-0.56$ & 110 & 2.2 & 1 & 5.5 & 2.2 & 1 \\
\hline G.E.Bulman ${ }^{13}$ & $2.2-6.25$ & 0.2994 & 0.6848 & 1.6 & 0.215 & 0.657 & 1.75 \\
\hline Bulman et al. ${ }^{14}$ & $6.25-2.22$ & 0.1899 & 0.575 & 1.82 & 0.2215 & 0.675 & 1.75 \\
\hline
\end{tabular}

\section{DESIGN CONSIDERATION}

The applications of IMPATT diode are mostly realized on the basis of double drift region and single drift region structures. However, the authors have considered a single drift region (SDR) IMPATT diode structure with doping distribution of the form $n^{+} n p^{+}$and $\mathrm{p}^{+} \mathrm{pn}^{+}$for the dc analysis[15]. The $\mathrm{n}^{+}$and $\mathrm{p}^{+}$regions of the diode are heavily doped with each having a doping concentration as mention in Table II. The 1-D schematic diagram of the proposed $\mathrm{p}^{+} \mathrm{nn}^{-} \mathrm{n}^{+}$High - Low SDR IMPATT diode is shown in Fig.3. 
Table II. Structural parameters of GaAs Hi-Lo SDR IMPATT Diode as [15]

\begin{tabular}{llllll}
\hline $\begin{array}{l}\text { High-Low } \\
\text { Structures }\end{array}$ & $\begin{array}{l}\text { High-region } \\
\text { Thickness }\end{array}$ & $\begin{array}{l}\text { High-region } \\
\text { Carrier } \\
\text { concentration } \\
\left(\mathrm{cm}^{-3}\right)\end{array}$ & $\begin{array}{l}\text { Low-region } \\
\text { Thickness }\end{array}$ & $\begin{array}{l}\text { Low-region } \\
\text { Carrier } \\
\text { concentration } \\
\left(\mathrm{cm}^{-3}\right)\end{array}$ & $\begin{array}{l}\text { Active region } \\
\text { Thickness } \\
(\mu \mathrm{m})\end{array}$ \\
$\mathrm{S}_{1}$ & 0.3 & $1 \times 10^{17}$ & 5.2 & $4.0 \times 10^{13}$ & 5.5 \\
$\mathrm{~S}_{2}$ & 0.45 & $1 \times 10^{17}$ & 5.05 & $3.5 \times 10^{15}$ & 5.5 \\
\hline
\end{tabular}

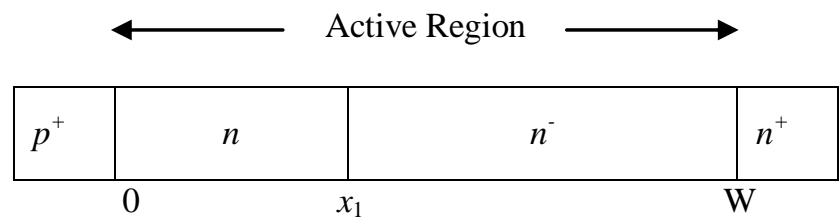

Fig.3 A 1-D schematic diagram of the proposed $\mathrm{p}^{+} \mathrm{nn}^{-} \mathrm{n}^{+}$High - Low SDR IMPATT diode.

\section{COMOUTOR SIMULATION METHOD}

The simulation method follows the DC analysis described in details elsewhere [21]. The equations involved in this analysis are nonlinear in nature and hence the solutions of these equations are quite complex. Therefore the authors have first considered the diode to be consisting of small space points. The diode active layer is divided into exactly 5000 space points with a space step of $1 \mathrm{~nm}$. In the steady state DC analysis, three important device equations namely Poisson's equation, the carrier continuity equation and the space charge equation have been solved simultaneously at each space point of the active layer of the diode.

Poisson's equation is given by,

$$
\frac{\partial E}{\partial x}=\frac{q}{\in}\left(N_{D}-N_{A}+p-n\right),
$$

The carrier continuity equation for electron and hole are given by,

$$
\begin{aligned}
& \frac{\partial n}{\partial t}=\frac{1}{q} \frac{\partial J_{n}(x)}{\partial x}+g(x) \\
& \frac{\partial p}{\partial t}=-\frac{1}{q} \frac{\partial J_{P}(x)}{\partial x}+g(x)
\end{aligned}
$$

where the avalanche carrier generation rate $(g)$ for electrons and holes is given by,

$$
g(x)=\alpha_{n}(x) n(x) v_{n}(x)+\alpha_{p}(x) p(x) v_{p}(x)
$$

For static condition equations, $(2 \& 3)$ can be written as:

$$
\frac{1}{q} \frac{\partial J_{p}(x)}{\partial x}=g(x)=-\frac{1}{q} \frac{\partial J_{n}(x)}{\partial x}
$$

Now using the relation $J_{p}=q v_{n} n$, the steady state carrier continuity equations (4) can be written as:

$$
\frac{\partial J_{p}(x)}{\partial x}=\alpha_{n} J_{n}(x)+\alpha_{p} J_{p}(x)=-\frac{\partial J_{n}(x)}{\partial x}
$$

Using the expression for total current density as $J=J_{n}+J_{p}=$ constant and defining,

$$
P(x)=\frac{J_{p}(x)-J_{n}(x)}{J}
$$

The pair of equations in (5) will reduce to the combined carrier continuity equation, 


$$
\frac{\partial P(x)}{\partial x}=\left(\alpha_{p}+\alpha_{n}\right)+\left(\alpha_{p}-\alpha_{n}\right) P(x)
$$

and the space charge equation, which gives the mobile space-charge concentration $(p-n)$ at any space point, can be obtained from equations (5) as,

$$
q \frac{\partial(p-n)}{\partial x}=J\left(\frac{\alpha_{n}}{v_{p}}+\frac{\alpha_{p}}{v_{n}}\right)-q\left(\alpha_{n}-\alpha_{p}\right)(p-n)+K \frac{\partial E}{\partial x}
$$

where $K$ is a correction factor whose value depends on the nature of the carrier velocity $\sim$ field characteristics in a semiconductor. For electrons and holes in silicon with velocity field relation:

$$
v_{n, p}=v_{s n, s p}\left[1-\exp \left(-\mu_{n, p} E / v_{s n . s p}\right)\right\rfloor
$$

The correction factor is found to be,

$$
K=\frac{J_{p} \mu_{p}}{v_{p}}\left(\frac{1}{v_{s p}}-\frac{1}{v_{p}}\right)-\frac{J_{n} \mu_{n}}{v_{n}}\left(\frac{1}{v_{s n}}-\frac{1}{v_{n}}\right)
$$

The above velocity field relation (Eq.8) is also valid for holes in III-V compound semiconductors e.g GaAs which is considered as the base material for this paper. However for electrons, the fourth power law $V \sim E$ relation is applicable for GaAs:

$$
v_{n}=\frac{\mu_{n} E+v_{s n}\left(E / E_{c}\right)^{4}}{1+\left(E / E_{c}\right)^{4}}
$$

The correction factor for velocity-field relation (10) is found to be,

$$
K=\frac{J_{p} \mu_{p}}{v_{p}}\left(\frac{1}{v_{s p}}-\frac{1}{v_{p}}\right)-\frac{J_{p}\left\{3\left[1-\frac{v_{n}}{v_{n}}\right]\left(E / E_{c}\right)^{4}-1\right\}}{v_{n} E\left[1+\left(E / E_{c}\right)^{4}\right]}
$$

Equations (1), (6) and (7) are now solved simultaneously to obtain the electric field and the carrier current profiles of the diode, subject to the boundary conditions obtained as follows. At the left edge of the depletion layer, the hole current comprises of only the reverse saturation current $J_{s p}$, which enters the depletion layer at this point. A double iterative DC simulation program is employed for this purpose. It iterates over the value of the electric field maximum $\left(\mathrm{E}_{\mathrm{m}}\right)$ and its location near the $n-p$ junction or the depletion layer of the diode subject to the usual boundary conditions.

The boundary conditions for the electric field at the edges of depletion layer are given by, $E(0)=0$ and $E(W)=0$ again the other boundary conditions for normalized current density $\mathrm{P}(\mathrm{x})$, are given by, $P(0)=\frac{2}{M_{p}}-1$ and $P(W)=\frac{2}{M_{n n}}-1$ where, $M_{n}=\frac{J}{I_{n s}}$ and $M_{n}=\frac{J}{J_{p s}}$ are hole and electron current multiplication factors, respectively, where $J_{p s}$ and $J_{p s}$ are electron and hole saturation current densities, respectively. The DC electric field profiles, carrier current profiles, breakdown voltage etc are obtained from this analysis.

\section{RESULT AND DISCUSSIONS}

The breakdown voltage of SDR High-Low (Hi-Lo) GaAs IMPATT diode has already been measured on different frequency of operation by Nisitani et al.[15]. Therefore, in this work, we have considered two different GaAs Hi-Lo SDR structures of IMPATT diodes as taken by Nisitani et al [15] and the breakdown voltages are determined using the computer simulation method as described earlier. The one dimensional structure of the proposed GaAs High-Low IMPATT diode is shown in figure-3 and the structural parameters are presented in Table II. The purpose of this paper is to ascertain the validity of the eight sets of ionization rate data by applying them to determine the breakdown voltage of high-low (HL) type GaAs avalanche transit time diodes, and to compare the results with available experimental data. The computed breakdown voltages have been compared with the experimentally measured breakdown voltage [15]. The computed values of the breakdown voltage of the different Hi-Lo SDR structures of GaAs diode using eight sets of ionization rates, have been presented in Table III. along with the experimentally determined breakdown voltages. 
Table III. Comparison of computed Breakdown voltages with experimentally determined values

\begin{tabular}{|c|c|c|c|c|c|c|c|c|c|}
\hline \multirow{2}{*}{$\begin{array}{l}\text { High-Low } \\
\text { SDR } \\
\text { structures }\end{array}$} & \multirow{2}{*}{$\begin{array}{l}\text { Experimentally } \\
\text { measured breakdown } \\
\text { voltages }\end{array}$} & \multicolumn{8}{|c|}{$\begin{array}{l}\text { Calculated Breakdown voltages from ionization data presented in } \\
\text { Table I. }\end{array}$} \\
\hline & & 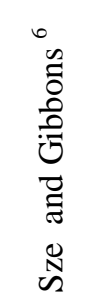 & 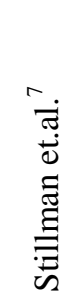 & 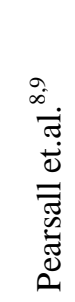 & 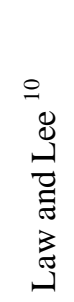 & 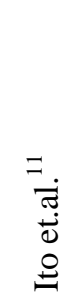 & 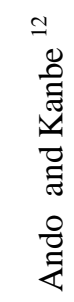 & 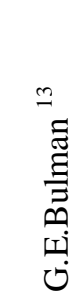 & 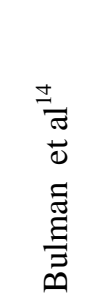 \\
\hline $\mathrm{S}_{1}$ & 19.5 & 4.3 & 7.06 & 19.8 & 6.45 & 22.5 & 14.8 & 13.3 & 9.38 \\
\hline $\mathrm{S}_{2}$ & 13 & 10.5 & 6.98 & 13.2 & 6.42 & 13.4 & 2.05 & 10 & 8.24 \\
\hline
\end{tabular}

It is evident that for High-Low structures $\left(S_{1}\right.$ and $\left.S_{2}\right)$, the computed breakdown voltage using the data of Pearsall et al. is very close to the experimentally determined breakdown voltage. On the other hand, for identical structures, the breakdown voltage computed from the data of Stillman et al., G.E.Bulman, Law and Lee, Robbin and Bulman, Ando and Kanbe and S.M.Sze et al. are less than the experimentally determined breakdown voltages. Again the breakdown voltages determined from the data of Ito et al. is greater than the experimentally measured breakdown voltage. Hence for the structure $S_{1}$ the breakdown voltage computed by using Pearsall et al. $[8,9]$ data is very close to the experimentally determined breakdown voltage i.e. $19.5 \mathrm{~V}$. The computed breakdown voltage using the ionization rate data of Pearsall et al. for structure $S_{2}$ is $13.2 \mathrm{~V}$, which is again very close to the experimentally determined breakdown voltage of $13 \mathrm{~V}$.

\section{CONCLUSION}

The study on carrier ionization rates of electron and hole in GaAs with reference to IMPATT diode has been carried out by using a computer simulation method as described earlier. Different sets of ionization rates given by various authors are considered in simulation method and the breakdown voltages of GaAs High-Low doping profile IMPATT diodes are computed using these parameters. The results thus obtained are compared with the experimental breakdown voltage. It is observed that the ionization rate data of GaAs reported by Pearsall et al $[8,9]$ is consistent with the breakdown voltage measurements of GaAs avalanche transit time diodes. Thus the carrier ionization rate reported by Pearsall et al may be considered while analyzing GaAs IMPATT diode.

[1] H Eisele, Electronics Lett. vol. 26, 1990, pp. 109.

\section{REFERENCES}

[2] H Eisele and G I Haddad, Electronics Lett. vol. 28, 1992, pp. 2176.

[3] S R Pattanaik, J K Mishra and G N Dash, IETE Journal of Research, vol. 57, 2011, pp. 351.

[4] P De, International conference on Microwave and Millimeter wave Technology, ICMMT-07, 2007, pp. 1-4.

[5] P R Tripathy, A K Panda and S P Pati, International Conference on Computers and Devices for Communication, CODEC- 09, 2009, pp. 1-4,

[6] S M Sze and G. Gibbons, App. Phys. Lett., vol. 8, 1966, pp. 111

[7] G E Stillman, C M Wolfe, J A Rossi and A G Foty, Appl. Phys. Lett., vol. 24, 1974, pp. 471.

[8] T Pearsall, F Capasso, R E Nahory, M A Pollack and J R Chelikowsky, Solid State Electronic, vol. 21,1978, pp. 297.

[9] T P Pearsall, R E Nahory, J R Chelikowsky, Phys. Rev. Lett., vol. 39, 1977, pp. 295,

[10] H D Law and C A Lee, Solid state Electron., vol. 21, 1978, pp. 331.

[11] M Ito, S Kagawa, T Keneda and T Yamaoka, J. Apply. Phys., vol. 49, 1978, pp. 4607,

[12] H Ando and H Kanbe, Solid State Electron., vol. 24, 1981, pp. 629.

[13] G E Bulman, V M Robbins, G E Stillman, K Hess and K F Brennan, IEEE Trans. Electron Devices Lett., vol. EDL-4,1983, pp. 181 .

[14] G E Bulman, V M Robbins and G E Stillman, IEEE Trans. Electron Devices, vol. ED-32, 1985, pp. 2454.

[15] K Nisitani, H Sawano, O Ishihari, T Ishii and S Mitsui, IEEE Trans. Electron Devices, vol. ED- 26, 1979, pp. 210.

[16] R A Logan, A G Chynoweth and B G Cohen, Phys. Rev., vol. 128, 1962, pp. 2518.

[17] R A logan and S M Sze, in Proc. Int. Conf. Phys. Semicond. (Kyoto, 1966), Suppl. J. Phys. Soc. Japan, vol. 21, 1966. pp. 434.

[18] M H Woods, W C Jhonson and M A Lampert, Solid State Electronic, vol. 16, 1973, pp. 381.

[19] F Capasso, R E Nahory, M A Polaack and T P Pearsall, Phys. Rev. Lett., vol. 39, 1977, pp. 723.

[20] F Capasso, R E Nahory and M A Polaack, Electron. Lett., vol. 15, 1979, pp. 117.

[21] G N Dash and S P Pati, Semicond. Sci. Tecnology, vol. 7, , 1992, pp. 222. 